\title{
Time-resolved sphere and fluid motions in turbulent boundary layers
}

\author{
Yi Hui Tee ${ }^{1 *}$, Ellen K. Longmire ${ }^{1}$ \\ ${ }^{1}$ University of Minnesota, Aerospace Engineering and Mechanics, Minneapolis, USA \\ *teexx010@umn.edu
}

\begin{abstract}
This paper extends the study by Tee et al. (2020) to investigate the effect of large coherent structures on motion of spheres with specific gravities of $1.006(\mathrm{P} 1)$ and $1.152(\mathrm{P} 3)$ at $R e_{\tau}=670$ and $1300\left(d^{+}=56\right.$ and 116). The sphere and fluid motions are tracked simultaneously via 3D particle tracking and stereoscopic particle image velocimetry over the streamwise-spanwise plane, respectively. With sufficient mean shear, sphere P1 lifts off of the wall upon release before descending back towards the wall at both $R e_{\tau}$. It typically accelerates strongly over a streamwise distance of less than one boundary layer thickness before approaching an approximate terminal velocity. By contrast, the denser sphere P3 does not lift off upon release but mainly slides along the wall. At lower $R e_{\tau}$ where wall friction is stronger, this sphere translates with unsteady velocity, significantly lagging the local fluid. The streamwise velocities of both spheres correlate strongly with the fast- and slow-moving zones that approach and move over them. In most runs, both spheres lag the local coherent structures and travel with either fast- or slow-moving zones throughout the observed trajectories. Vortex shedding, which is most prevalent for sphere P3 at $R e_{\tau}=670$, is also important. The sphere spanwise motion is prompted by wall friction, spanwise fluid motion, and/or meandering of the coherent structures, and spheres do not appear to migrate preferentially into slow-moving zones.
\end{abstract}

\section{Introduction}

In particle-laden flow, the transport of particles such as sand, dust, sediment, plankton, and pollutants in air, rivers, or oceans is complicated by the presence of particle-wall and particle-turbulence interactions. As these flows are turbulent in nature, coherent structures in the boundary layer can have significant effects on particle suspension, deposition, and transport. Early studies examining particle wall-normal motion in turbulent open-channel flows include imaging experiments from Sutherland (1967), Francis (1973), and Sumer and Oguz (1978), to name a few. Sutherland (1967) proposed an entrainment hypothesis whereby strong turbulent eddies could disrupt the viscous sublayer and lift grains off of an underlying sediment bed. Later, Kaftori et al. (1995), van Hout (2013), and Baker and Coletti (2021), among others, incorporated direct visualization techniques to measure particle and fluid velocities. These studies concluded that particle resuspension and deposition events are strongly influenced by the coherent structures and intermittent fluid forces acting on the particle. Research by Rashidi et al. (1990), Pedinotti et al. (1992), and Niño and Garcia (1996) on particle spanwise motion concluded that small inertial particles with diameter, $d^{+} \sim O(1)$ tend to accumulate in low-speed streaks near the wall where ejection events are prominent. From here onward, the superscript + denotes quantities normalized by the friction velocity $\left(u_{\tau}\right)$ and the kinematic viscosity $(v)$.

Particles of finite size can experience variations in shear and normal forces around their circumferences. In this context, the study by Costa et al. (2020) comparing the results between interface-resolved and one-way-coupled point-particle direct numerical simulations (DNS) demonstrated distinctive differences in particle behavior near the wall due to the absence of any shear-induced lift force in the point-particle model. This finding also highlights the importance of instantaneous fluid forces on the motion of discrete particles. Zeng et al. (2008) quantified the fluid forces acting on a fixed sphere located at multiple distances above a smooth wall. The mean lift forces simulated for spheres with $1.78 \leq d^{+} / 2 \leq 12.47$ centered at a wallnormal location $y^{+}=17.31$ from the wall at friction Reynolds number, $R e_{\tau}=178.12$ were negative in all cases. Tomographic PIV performed by van Hout et al. (2018) at $R e_{\tau}=352$ downstream of a tethered sphere with $d^{+} / 2=25$ centered at $y^{+}=43$ above the wall also suggested a negative lift contribution due to the 
sphere wake tilting away from the wall. By contrast, Hall (1988), who measured the mean lift force acting on a stationary particle lying on the wall in a turbulent boundary layer using a force transducer, reported a positive lift contribution. The experimental data showed that for $3.6<d^{+}<140$ and particle Reynolds number, $6.5<R e_{p}<1250$, the normalized mean lift forces are strongly positive and could be approximated by $F_{L}^{+}=(20.90 \pm 1.57)\left(d^{+} / 2\right)^{2.31 \pm 0.02}$. These results imply that the net wall-normal force including lift related to the mean shear can vary significantly with the sphere position relative to the wall. All of these effects can significantly impact particle motion in wall-bounded turbulent flows, where the particles can either slide or roll along the bounding surface or lift off from and collide with the wall.

Aside from lift forces induced by mean shear and turbulence, Magnus lift can play a role when solid body rotation is present. This effect remains relatively unstudied in particle-laden turbulent wall-bounded flow due to the challenges in reconstructing and modeling particle rotation. Current experimental methods for reconstructing sphere orientation include printing specific patterns over the sphere surface (Zimmermann et al., 2011; Mathai et al., 2016) and embedding visible tracers into the interior of transparent spheres (Bellani et al., 2012; Klein et al., 2013). The former method compares the unique pattern captured by high-speed cameras with synthetic projections to extract the absolute sphere orientation; the latter method resolves tracer velocities within the solid body to obtain the rotation rate. Barros et al. (2018) extended Klein et al.'s (2013) methodology to include opaque spheres. Small dots were marked all over the solid surface, and two cameras were employed to reconstruct the 3D rotation rate using Kabsch's (1976) algorithm.

In the present study, we conduct simultaneous particle tracking and stereoscopic particle image velocimetry (SPIV) to investigate the 3D motion of individual finite-size spheres and the surrounding fluid motion in turbulent boundary layers. Multiple sphere densities and flow conditions are considered. To obtain both the translation and rotation of a sphere, Barros et al.'s (2018) methodology is adapted to the requirements of the current experimental setup. A detailed discussion on sphere motion has been reported previously in Tee et al. (2020). In this paper, we will focus on the new results that we have obtained on fluid motion surrounding a moving sphere to understand particle-turbulence and particle-wall interactions.

\section{Methodology}

The experiments were conducted in a recirculating water channel with a glass test section of $8 \mathrm{~m}$ length and $1.12 \mathrm{~m}$ width. A $3 \mathrm{~mm}$ cylindrical trip-wire located at the entrance of the test section triggered the development of a turbulent boundary layer along the bottom wall. We focus on $R e_{\tau}=670$ and 1300 to investigate the effect of mean shear and turbulence on sphere motion. The water depths were maintained at $0.396 \mathrm{~m}$ and $0.392 \mathrm{~m}$ respectively. These correspond to free-stream velocities $\left(U_{\infty}\right)$ of 0.205 and $0.464 \mathrm{~m} \mathrm{~s}^{-1}$, with boundary layer thicknesses $(\delta)$ of 0.076 and $0.073 \mathrm{~m}$. The mean flow statistics of the unperturbed turbulent boundary layers were determined from planar PIV measurements in streamwise wall-normal planes. Hereafter $x, y$, and $z$ define the streamwise, wall-normal, and spanwise directions respectively.

To achieve a repeatable and controllable initial condition, magnetic spheres molded from a mixture of wax and iron oxide were used. The sphere surfaces were black, and white dots were painted at arbitrary locations using an oil-based pen to monitor both translation and rotation (see inset in Figure 1p). We consider two spheres of diameter, $d=6.35 \mathrm{~mm}$ with specific gravities $\left(\rho_{p} / \rho_{f}\right)$ of $1.006(\mathrm{P} 1)$ and $1.152(\mathrm{P} 3)$, where $\rho_{p}$ is the sphere density and $\rho_{f}$ is the fluid density. The sphere diameters are significantly larger than the Kolmogorov length scale, with $d^{+}$of 56 and 116 respectively. Meanwhile, the particle Stokes numbers $\left(S t^{+}, S t_{\delta}\right)$ expressed as the ratio of particle response time, $\tau_{p}=\left(\rho_{f}+2 \rho_{p}\right) d^{2} / 36 v \rho_{f}$ (Crowe, 2005) to the characteristic flow time scale based on viscous time scale $\left(v / u_{\tau}^{2}\right)$ and largest time scale $\left(\delta / U_{\infty}\right)$, range from 262 to 1230 and 9.1 to 23.5 , respectively. The initial particle Reynolds numbers defined as $R e_{p}=U_{\text {rel }} d / \mathrm{v}$ are 730 and 1730, where $U_{\text {rel }}$ is the difference between the mean fluid velocity and velocity of the particle center. For each run, a given sphere was held statically on the smooth wall in the boundary layer by a magnet placed flush with the outer wall of the channel. The sphere was positioned at a location $4.2 \mathrm{~m}$ downstream of the trip wire and $4 \delta$ away from the nearest sidewall. This location will be considered as the origin in $x$ and $z$, with the bottom wall as $y=0$. The magnet was then deactivated, releasing the sphere and allowing it to propagate with the incoming flow. A screen was located at the end of the test section to capture the sphere and prevent it from recirculating around the channel. For each case considered, up to $J=10$ trajectories were captured using the same sphere. Details of the experimental parameters are summarized in Table 1 .

For sphere tracking, two pairs of Phantom v210 high-speed cameras were arranged in stereoscopic configurations, looking from the side wall, to track the sphere in 3D space over a relatively long field of view (see Figure 19). Two white LED panels illuminated the domain considered. The particles were tracked over a streamwise distance up to $x=5 \delta$. Before computing the particle translation and rotation, the gray- 
(a) Top View

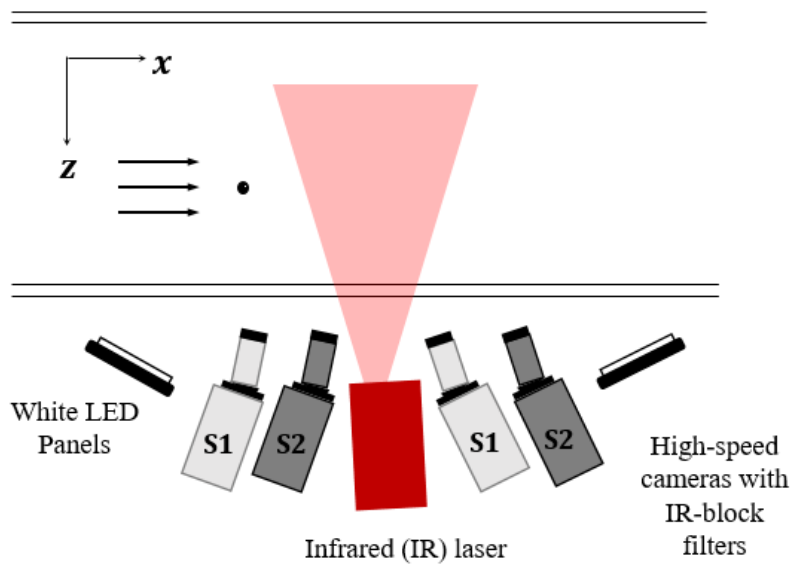

(b) Cross-section View

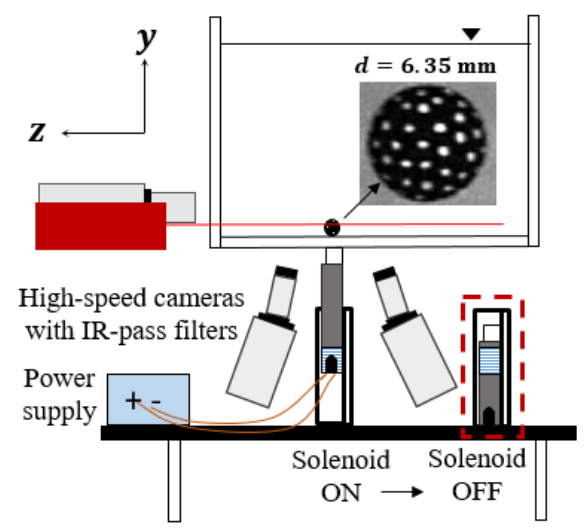

Figure 1: Experimental setup. (a) Top view: two pairs of high-speed cameras (S1 and S2) with infrared (IR) -block filters, aligned in stereoscopic configurations for capturing the trajectory and rotation of a marked sphere over a long field of view. (b) Cross-section view: a pair of stereoscopic high-speed cameras with IR-pass filters, positioned under the channel for capturing the fluid motion illuminated by the infrared laser over the streamwise-spanwise plane. The sphere was held in place by a magnet connected to a solenoid $(\mathrm{ON})$. Dashed line box illustrates the magnet position after turning off the power supply (solenoid OFF) to release the sphere. Inset: example of sphere captured in gray-scale with diameter $(d)$ spanning $\sim 43$ pixels.

Table 1: Summary of experimental parameters. $\left|V_{s}\right|$ represents sphere settling velocity magnitude in quiescent flow; ${\overline{F_{L}}}^{*}=\left(\overline{F_{L}}-F_{b}\right) / F_{b}$ where $\overline{F_{L}}$ and $F_{b}$ denote the mean wall-normal fluid-induced force based on Hall's (1988) expression and the net buoyancy force, respectively.

\begin{tabular}{ccccccccc}
\hline$R e_{\tau}$ & Initial $R e_{p}$ & $d^{+}$ & Sphere & $\rho_{p} / \rho_{f}$ & $\left|V_{s}\right| / U_{\infty}$ & $S t^{+}$ & $S t_{\delta}$ & Initial $\bar{F}_{L}{ }^{*}$ \\
\hline 670 & 730 & 56 & $\mathrm{P} 1$ & 1.006 & 0.083 & 262 & 9.10 & $11 \pm 2$ \\
& & & $\mathrm{P} 3$ & 1.152 & 0.78 & 287 & 9.98 & $-0.77 \pm 0.04$ \\
\hline \multirow{2}{*}{1300} & \multirow{2}{*}{1730} & \multirow{2}{*}{116} & $\mathrm{P} 1$ & 1.006 & 0.037 & 1120 & 21.4 & $61 \pm 10$ \\
& & & $\mathrm{P} 3$ & 1.152 & 0.34 & 1230 & 23.5 & $0.24 \pm 0.2$ \\
\hline \hline
\end{tabular}

scale images were pre-processed using a Matlab circular Hough Transform routine to isolate the sphere from the background. The extracted sphere images were then imported to Davis 8.4 and further processed with 3 x 3 Gaussian smoothing and sharpening routines to increase the dot contrasts. Subsequently, pixel intensity values that were less than the white dots were set to 0 to isolate the dots from the sphere image. After performing a volumetric calibration, a 3D-PTV routine was implemented to reconstruct the dot coordinates from both camera pairs. The data sets obtained from PTV were composed of the 3D coordinates of true and ghost markers and their corresponding 3D velocity vectors. Hence, the filtering methodology proposed by Barros et al. (2018) was employed to remove the ghost tracks. Once the true markers were identified, the sphere centroid was determined by applying the equation of a sphere. Then, a rotation matrix that best aligned the markers of consecutive images was obtained (Barros et al., 2018).

For fluid motion, a third pair of high-speed cameras (Phantom M110) was arranged in stereoscopic configuration, looking from the bottom wall, to obtain the three components of fluid velocity within a $x-z$ plane (see Figure 1 $\mathrm{b}$ ). The flow was seeded with silver-coated hollow glass sphere tracers, with an average diameter and density of $13 \mu \mathrm{m}$ and $1600 \mathrm{~kg} \mathrm{~m}^{-3}$, respectively. An Oxford Firefly infrared pulsed laser with a wavelength of $808 \mathrm{~nm}$ was used to illuminate the tracer particles. The laser sheet illuminated through the side wall at $y=0.7 d$ had a thickness of $1 \mathrm{~mm}$ with field of view of $0.3<x / \delta<1.7$ and $-0.5<z / \delta<$ 0.5 . Infrared-block and -pass optical filters were mounted to the lenses of the tracking and SPIV cameras respectively to optimize the image quality. To capture both time-resolved sphere and fluid motions, all six cameras were triggered simultaneously with the infrared laser at 240 and $480 \mathrm{~Hz}$ for $\operatorname{Re}_{\tau}=670$ and 1300 , respectively. Stereoscopic self-calibration was carried out on top of the classic calibration using 200 image 
(a)

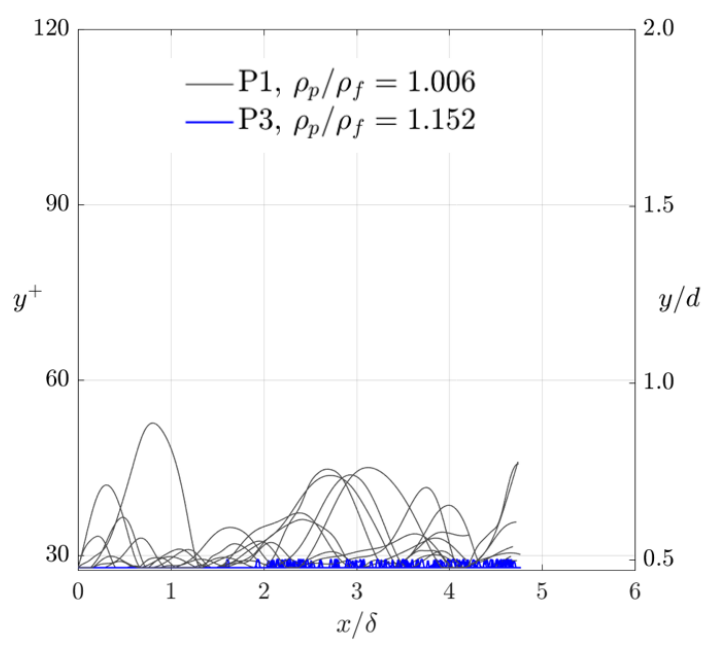

(b)

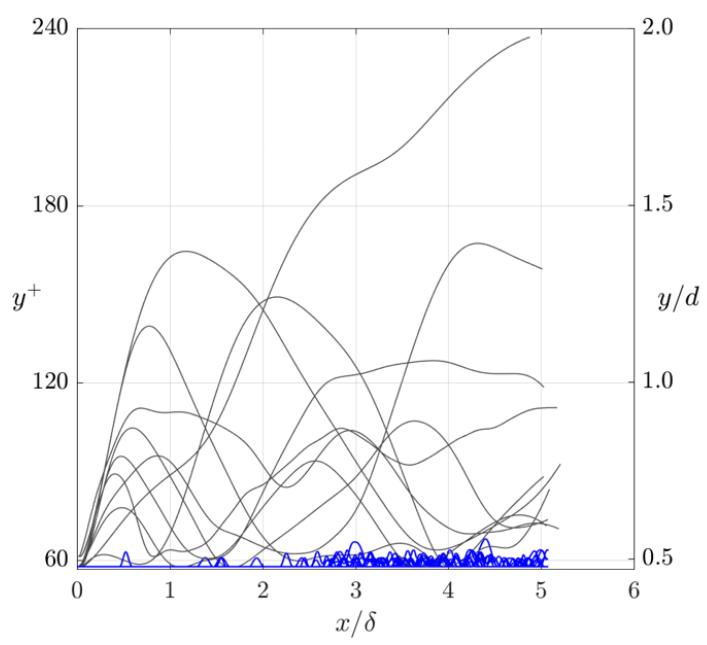

Figure 2: Sphere wall-normal trajectories $(y)$ plotted based on the centroid positions at $R e_{\tau}=670$ (a) and $R e_{\tau}=1300$ (b), respectively. Left axis: normalized by inner scaling; Right axis: normalized by sphere diameter.

pairs from unperturbed flow fields. Prior to processing the SPIV images in Davis 10.1, the sphere, which appeared as a very bright spot, was removed using Matlab. Then, the spatial auto-mask function in Davis 10.1 was implemented to mask out regions without tracer particles such as the shadow and area where the sphere had been extracted. Sliding sum-of-correlation with a filter length of 2 images, and an overlap of $50 \%$ over initial interrogation window sizes of 64 by 64 pixels followed by three passes of 32 by 32 pixels was then employed to obtain the three-component velocity vectors (see Sciacchitano et al., 2012). Spurious vectors were removed based on the universal outlier detection criterion (Westerweel and Scarano, 2005). The spatial resolutions of the computed velocity vectors are 24 and 50 viscous units at $R e_{\tau}$ of 670 and 1300 , equivalent to $2.73 \mathrm{~mm}$. The mean unperturbed fluid velocities $(\bar{U})$ at laser sheet heights of $y^{+}=40$ and 80 are 0.124 and $0.287 \mathrm{~m} \mathrm{~s}^{-1}$, respectively.

\section{Results and Discussion}

\subsection{Sphere wall-normal motion}

Figure 2 shows the sphere wall-normal $(y)$ trajectories plotted against their streamwise distance traveled, with all particle runs superposed. Note that these trajectories represent new data obtained from the current simultaneous sphere and fluid tracking experiments, using two out of the three spheres considered in Tee et al. (2020). The sphere behavior was consistent with the previous runs. For completeness, we will provide a brief summary of the sphere motions here. Readers are encouraged to refer to Tee et al. (2020) for a more detailed analysis of three-dimensional sphere translation and rotation. For stationary spheres, the mean lift forces against the net buoyancy forces are computed using Hall's (1988) equation obtained for a fixed sphere in a turbulent boundary layer where $\overline{F_{L}}=F_{L}^{+} v^{2} \rho_{f}, F_{b}=\left(m_{p}-m_{f}\right) g$ and $\bar{F}_{L}{ }^{*}=\left(\overline{F_{L}}-F_{b}\right) / F_{b}$ (see Table 1 . Here, $m_{p}$ is the mass of sphere, $m_{f}$ is the mass of fluid displaced and $g$ is the gravitational acceleration. As all spheres are denser than water, the net buoyancy force will always act downward in the direction of gravity. Thus, if ${\overline{F_{L}}}^{*}>0$, the sphere is likely to lift off upon release, and vice versa.

For the least dense sphere P1, at $R e_{\tau}=670, \bar{F}_{L}{ }^{*}=11$. Increasing $R e_{\tau}$ to 1300 doubles $d^{+}$and thus the sphere mean lift force increases fivefold. These estimations agree very well with our observations where sphere P1 mostly lifts off of the wall once release at both $R e_{\tau}$ (plotted as black in Figure 2). Additionally, owing to the stronger resultant upward force, sphere $\mathrm{P} 1$ at $R e_{\tau}=1300$ lifts off higher than at $R e_{\tau}=670$. This shows that the initial lift-off height correlates strongly with the local mean shear. For the densest sphere P3, no initial lift-offs are observed (plotted as blue in Figure 2). This sphere does not have sufficient lift force to overcome the downward force and translates along the wall upon release. Here, the sphere slides but does not roll along the wall upon release, unlike what was observed by Drake et al. (1988) and Yousefi et al. 
(2020) for dense particles on rough beds. In general, most of our results on initial sphere lift-off agree well with the Hall's (1988) equation. By varying the initial turbulent conditions, Yousefi et al. (2020) concluded also that the mean flow irrespective of the initial turbulent structures is responsible for the particle lift-offs.

Sphere P1, which always lifts off initially, always descends towards the wall after reaching a local maximum in height, typically undergoing multiple subsequent lift-off events. This sphere either contacts the wall and then lifts off again, or else reascends without returning to the wall, similar to what was reported by Francis (1973), Sumer and Oguz (1978), van Hout (2013), and Yousefi et al.(2020). From our studies, we also noticed that even after touching and sliding along the wall, the sphere could ascend to greater heights than the initial maximum. At the lower $R e_{\tau}$, the sphere lifts off more frequently and to lower maximum heights. The lift-off angles are less than $12^{\circ}$ throughout all of the trajectories. For sphere P1, only minimal rotations are observed at both $R e_{\tau}$ throughout all trajectories.

Sphere P3, which does not generally lift off upon release, did experience repeated lift-off events further downstream, with magnitude of $\leq 0.1 d$. This behavior is observed frequently at both $\operatorname{Re}_{\tau}$ after the sphere begins to roll forward, occurring consistently starting at $x \approx 2 \delta$ when $R e_{\tau}=670$. The rotational velocity is stronger at $R e_{\tau}=670$ than at $R e_{\tau}=1300$, thus indicating a stronger forward rolling to sliding tendency.

\subsection{Sphere streamwise velocity}

Figures 3 and 4 illustrate the respective sphere $\mathrm{P} 1$ and $\mathrm{P} 3$ streamwise velocities $\left(U_{p}\right)$ at $R e_{\tau}=670$ for all runs (left axis; solid lines). The sphere velocities are normalized by the mean unperturbed fluid velocities at the height of the sphere centroids $(\overline{U(y)})$. In a uniform, steady, unbounded flow, an initially stationary sphere always accelerates to the local fluid velocity with zero relative velocity. In our studies, however, the presence of turbulence and the wall both modify the surrounding flow fields and sphere kinematics. Due to the large initial drag, the spheres accelerate strongly upon release until they achieve an approximate terminal velocity. This acceleration occurs within $x \leq 1 \delta$ for all cases except P3 at $R e_{\tau}=670$. The approximate terminal velocity for sphere $\mathrm{P} 1$ is closer to the mean local fluid value than that for $\mathrm{P} 3$ at both $R e_{\tau}$. Even though sphere P1 has density fairly close to that of the fluid, it still lags behind the local fluid mean in most runs with infrequent occasions where it travels faster than the local fluid mean at both $\operatorname{Re}_{\tau}$. For this sphere, the streamwise velocity fluctuation also correlates positively with the respective $y$-trajectory as shown by the two sample runs (red and blue curves) in Figure 3 a. Here, as sphere P1 ascends, it gains more momentum from the faster-moving fluid away from the wall and accelerates; as it descends, it loses momentum due to the slower-moving fluid near the wall and thus begins to decelerate.

Meanwhile, the velocity curves for sphere P3 at $R e_{\tau}=670$ initially fluctuate significantly with shorter wavelengths before increasing strongly near $x>1.5 \delta$ to an approximate terminal velocity. This delayed acceleration also corresponds with the times where the sphere transitions from forward sliding to rolling with small repeated lift-off events. As the small lift-off events $(x>2 \delta)$ occur at a much shorter wavelength than the corresponding streamwise velocity fluctuations in the same region, these variations appear decoupled as opposed to sphere P1. As sphere P3 interacts with the wall more often, its forward motion is strongly retarded by the friction force compared with sphere P1 which mostly translates above the wall.

After attaining an approximate terminal velocity, velocity fluctuations of order $\pm 0.2 U_{\infty}$ are observed in most runs for all cases. For sphere P1, as highlighted above, although the velocity fluctuation matches the sphere wall-normal position, the magnitude of the fluctuations is not directly proportional to the lift-off magnitude. Here, within the same case, the velocity curves also spread over a wide range compared with mean sphere velocity. This suggests that the velocity gradient that changes with the wall-normal position is not the only reason behind the sphere streamwise velocity fluctuation. To understand the widespread variation of the sphere streamwise velocity, we look at the corresponding streamwise fluid velocity contour plots. Within streamwise-spanwise planes of the logarithmic layer, long coherent structures that appear as alternating fast- and slow-moving zones, or high and low momentum regions, are observed commonly as previously reported by researchers such as Dennis and Nickels (2011) and Tan and Longmire (2017). Here, we notice that these structures have significant effects on the sphere streamwise velocity such that a sphere traveling in a low momentum region (Figure $3 \mathrm{~b}$ which corresponds to the blue $U_{p}$ curve in Figure $3 \mathrm{a}$ ) accelerates less and lags the mean flow more than the same sphere traveling in a high momentum region (see $U_{p}$ plotted in red in Figure $3 \mathrm{a}$ and the corresponding fluid velocity in Figure 3 r). Meanwhile, sphere P3 at the same $R e_{\tau}$ lags the fluid more significantly due to stronger wall friction. Therefore, it is overtaken repeatedly by both low and high momentum regions as shown in Figure $4 \mathrm{~b}$, causing its streamwise velocity to fluctuate more rapidly than that for sphere P1. As the spheres are significantly larger than the Kolmogorov length scale $(d=25$ and $44 \eta)$, and $S t^{+}$are significantly larger than $S t_{\delta}$, the spheres are more likely to be accelerated and decelerated by the large scale motions in the logarithmic region than by smaller scale motions closer to 
(a)

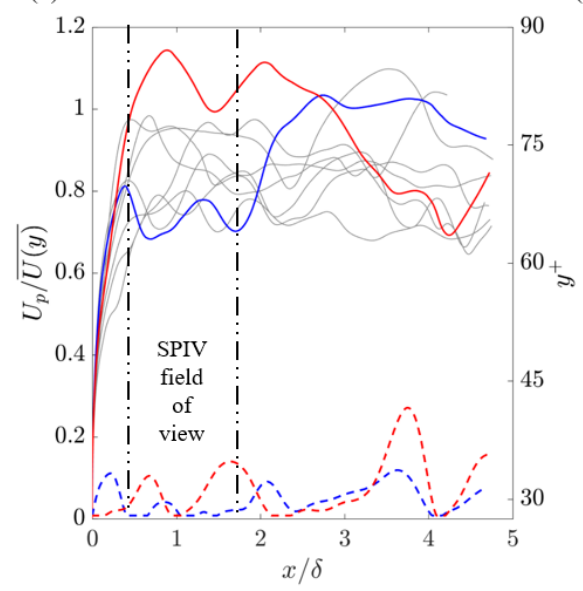

(b)

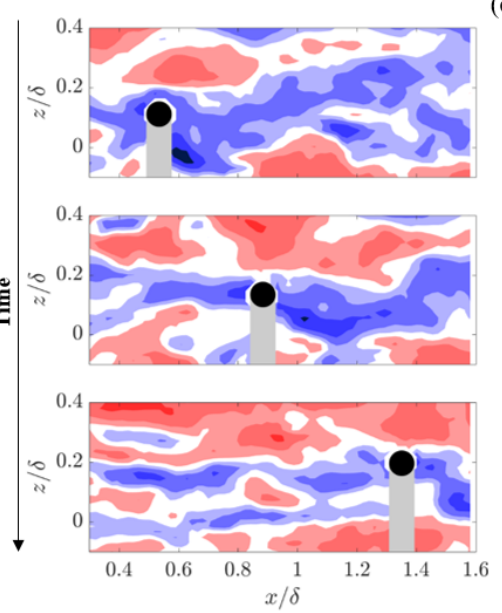

(c)

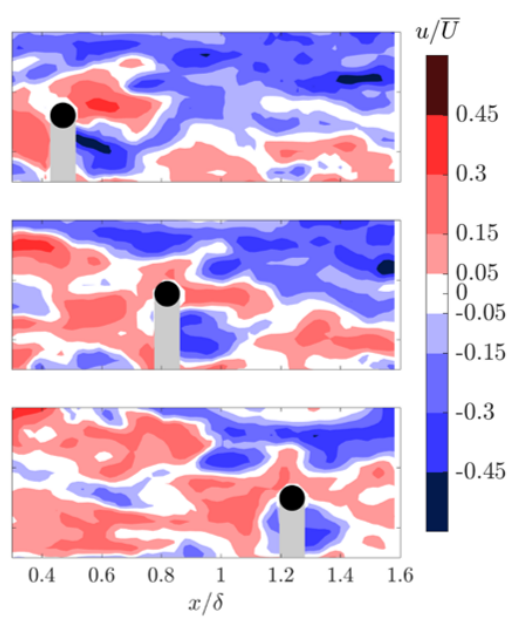

Figure 3: Sphere P1 at $R e_{\tau}=670$. (a) Left axis (solid lines): Sphere streamwise velocity, $U_{p}$ normalized by mean unperturbed fluid velocity at the sphere center location for all runs. Right axis (dashed lines): sphere wall-normal trajectories. Blue and red curves: P1 in low and high momentum regions, respectively. Black dash-dot lines: SPIV field of view. (b, c) Samples of time-resolved fluctuating streamwise fluid velocity, $u$ surrounding the sphere in low and high momentum regions (blue and red curves in (a)) respectively. $\bar{U}$ is mean unperturbed fluid velocity at the laser sheet height of $y^{+}=40(y=0.7 d)$. Grey region under the sphere represents sphere shadow.

the wall as reported by Ebrahimian et al. (2019).

In addition, distinct wake features can be observed consistently downstream of sphere P3 within the SPIV field of view as indicated by the regions with strong negative $u$ in Figure $4 \mathrm{~b}$. Vortex shedding can be identified from the black and green contour lines which represent clockwise and counter-clockwise swirls. The swirling strength is computed from the imaginary part of the complex eigenvalue of the local velocitygradient tensor and normalized by its root mean square value (see $\mathrm{Wu}$ and Christensen, 2006). Swirling regions including 4 or more grid points are then identified using a threshold of 0.5 . In the measurement plane, these appear as pairs of counter-rotating vortices, which could be slices of hairpin-like loops as observed by van Hout et al. (2018) using tomographic PIV. In this region, the local particle Reynolds numbers are $\sim O(500)$. As sphere P1 translates with velocity closer to the mean fluid value, its $R e_{p}$, after it accelerates steeply from rest, decreases from 730 and 1730 at $R e_{\tau}=670$ and 1300 to a range between $1<R e_{p}<300$. If we consider sphere P1 in Figure $3 \mathrm{~b}$, which translates relatively slower than the local fluid mean and where the instantaneous $\operatorname{Re}_{p}$ is about 220, then vortex shedding would be present occasionally based on Zeng et al.'s (2008) conclusion for $R e_{p}>200$. By contrast, for sphere P1 in Figure 3c, which translates faster than the local mean fluid and has an instantaneous $R e_{p}<50$, vortex shedding would typically be absent. Here, the region of slow moving fluid upstream of and below the sphere is most likely the wake left behind as the sphere accelerates from rest and moves in the positive $z$ direction. Therefore, for a discrete particle with significant $R e_{p}$, vortex shedding can play an important role not only in affecting the instantaneous drag force but also in modifying the turbulence organization. For particles very close to the wall, the shedding also likely affects the instantaneous wall-normal force.

To validate our findings on sphere velocity variation, we computed the two-point spatial correlation coefficients between the sphere and fluid velocities across all runs in both streamwise and spanwise directions within the SPIV field of view at $y=0.7 d$ using the following equation:

$$
R_{U_{p}, U_{f}}(x, z)=\frac{1}{J-1} \sum_{j=1}^{J}\left(\frac{U_{p}\left(x_{o}, z_{o}\right)-\overline{U_{p}\left(x_{o}, z_{o}\right)}}{\sigma_{U_{p}}}\right)\left(\frac{U_{f}\left(x_{o}+\Delta x, z_{o}+\Delta z\right)-\overline{U_{f}\left(x_{o}+\Delta x, z_{o}+\Delta z\right)}}{\sigma_{U_{f}}}\right)
$$

The over-line represents time-averaged quantity within the field of view in each run; subscript 'o' represents the origin for spatial correlation; $\sigma$ represents the standard deviation. In most runs, even though the spheres move in the $y$-direction (especially P1), they do not rise completely above the laser sheet. In the correlations, we consider only results where the sphere intersects the laser sheet positioned at $y=0.7 d\left(y^{+}=40\right.$ and 80 for $R e_{\tau}=670$ and 1300, respectively) throughout the SPIV field of view. The results for both P1 and P3 

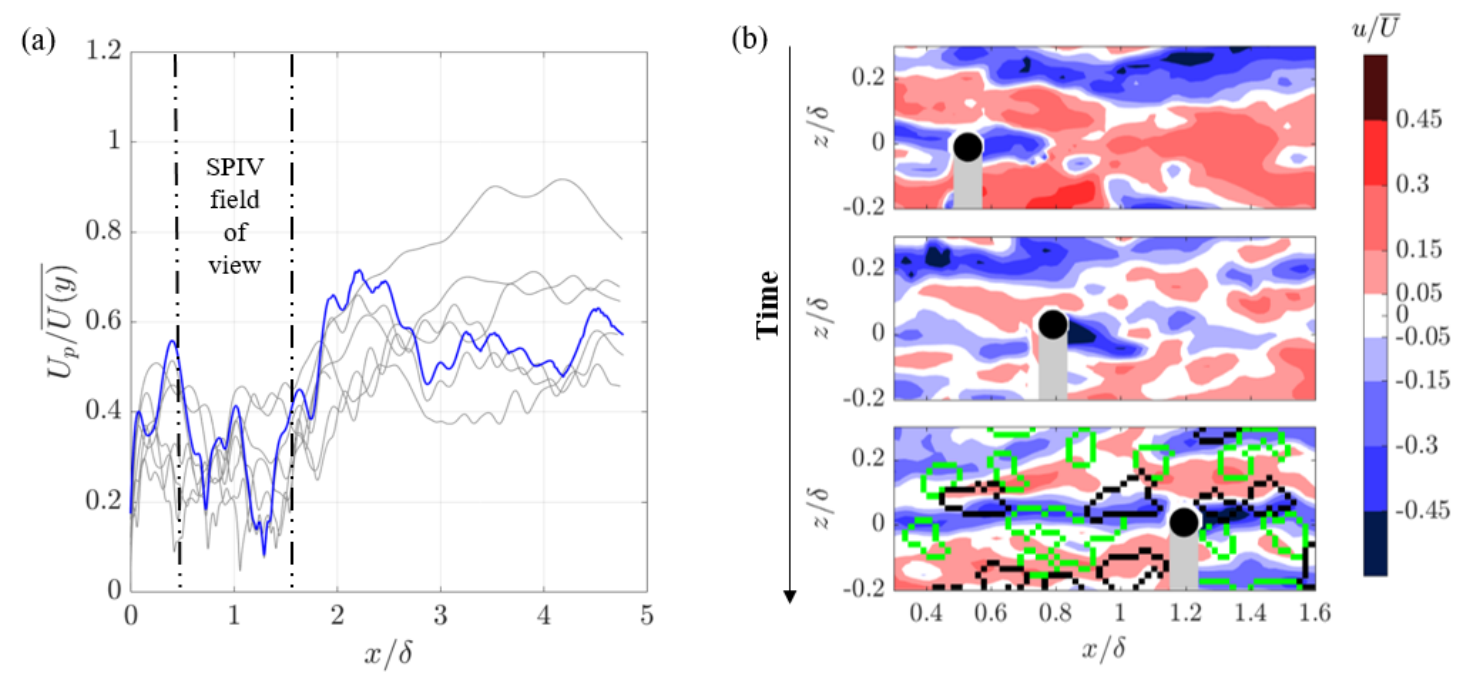

Figure 4: Sphere P3 at $R e_{\tau}=670$. (a) Sphere streamwise velocity, $U_{p}$ normalized by mean unperturbed fluid velocity at the sphere center location for all runs. Blue line: sample run in (b). Black dash-dot lines: SPIV field of view. (b) Sample of time-resolved fluctuating streamwise fluid velocity, $u$ surrounding the sphere plotted as blue in (a). Black and green contours in the bottom plot represent clockwise and counterclockwise swirls.

(a)

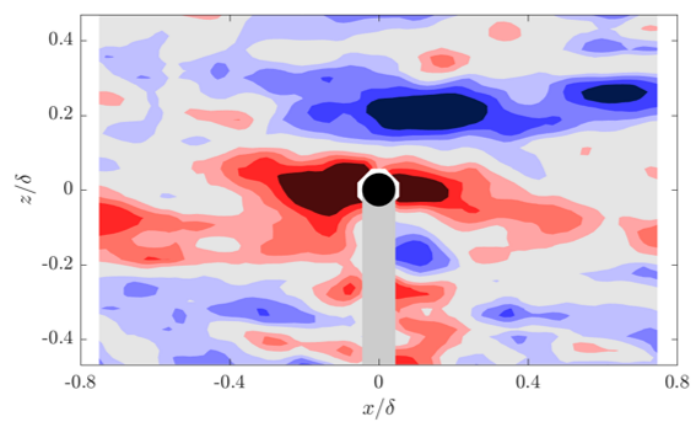

(b)

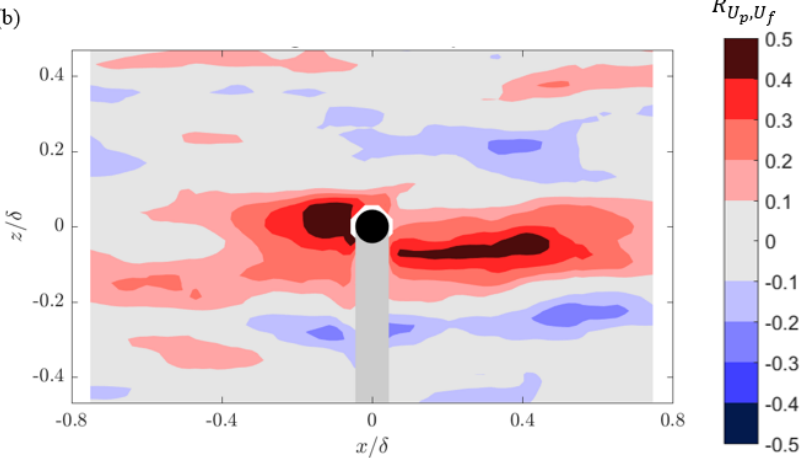

Figure 5: Two-point spatial correlation coefficients between the sphere and fluid streamwise velocities at $R e_{\tau}=670$ for sphere P1 (a) and P3 (b).

at $R e_{\tau}=670$ are plotted in Figure 5 in the form of contour plots, with the origin $\left(x_{o}, z_{o}\right)$ centered at the sphere centroid location. Note that even though the number of runs is insufficient to achieve smooth statistical convergence over the entire domain shown, the results are nevertheless useful in highlighting the predominant trend for large-scale particle-turbulence interaction. The results demonstrate that the streamwise velocity of these spheres is positively correlated with the surrounding streamwise fluid velocity $\left(R_{U_{p}, U_{f}}(x, z)>0\right.$; red). Specifically, the sphere velocity is strongly affected by the fast- and slow-moving zones that move over the sphere in the form of long, relatively narrow structures. Adjacent to the positively correlated region are long, narrow regions with negative correlation (blue) indicative of the surrounding fluid structures. Here, the sphere velocity is positively correlated with both the upstream and downstream fluid. For sphere P1, the upstream and downstream patterns have similar strength and size. By contrast, for sphere P3, the downstream region remains positively correlated over a longer distance, likely a result of the stronger wakes present in this case. These results imply that for an initially stationary sphere with large $R e_{p}$, upon release, its forward motion, which is governed by the relative force between the drag and friction, is strongly dependent on the coherent structures that approach and move over the spheres as well as the vortex shedding. For sphere $\mathrm{P} 1$, its wall-normal force, which is a function of the wall-normal component of the fluid velocity and the surrounding shear and pressure fields, must also be affected by these fluid velocity variations. 
(a)

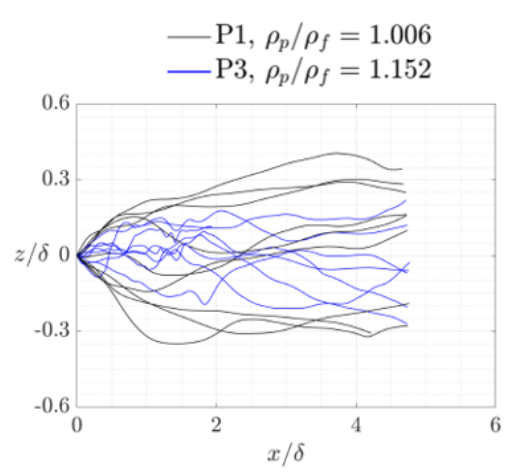

(b)

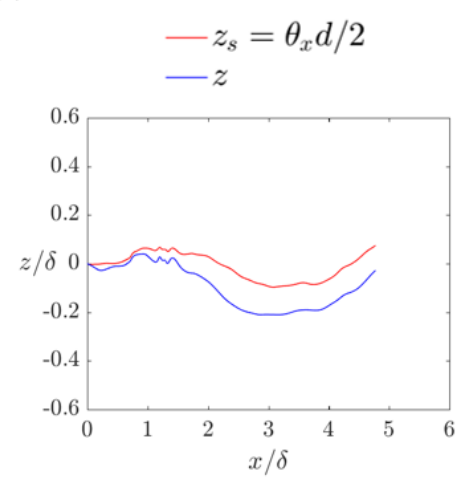

(c)

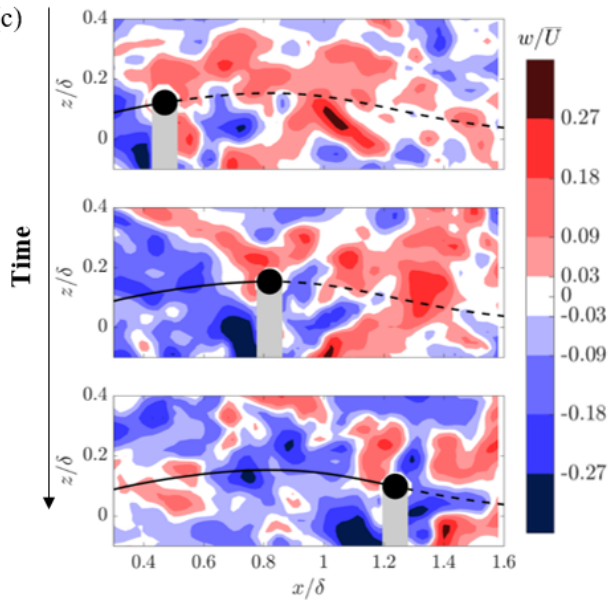

Figure 6: (a) Sphere spanwise positions plotted based on the centroid locations at $R e_{\tau}=670$ for sphere P1 (black) and P3 (blue). (b) Sample trajectory for sphere P3 at $R e_{\tau}=670$. (c) Sample of time-resolved spanwise fluid velocity, $w$ surrounding sphere P1 at $R e_{\tau}=670$, plotted in red in Figure 3 a. The black curves superposed on top of the contours represent the sphere spanwise position along its trajectory.

\subsection{Sphere spanwise motion}

The spanwise trajectories of spheres $\mathrm{P} 1$ and $\mathrm{P} 3$ at $R e_{\tau}=670$ are plotted in Figure 6a. Once released, instead of propagating along $z=0$, the spheres typically move sideways to some degree. Then, they either continue to propagate in one direction or else reverse and travel in the opposite direction, with some crossings over the $z=0$ plane. In all cases, the maximum spanwise distance traveled from $z=0$ is approximately $12 \%$ of the corresponding streamwise distance traveled.

For the wall-interacting sphere $\mathrm{P} 3$, most of the spanwise curves initially fluctuate at higher frequencies than in the other lifting cases. The range including the direction changes (when $x<2 \delta$ ) corresponds directly with the region prior to the onset of forward rolling. If we assume that the displacement results from a pure rolling motion about the streamwise axis, then the spanwise displacement based would be $z_{s}=\theta_{x} d / 2$. This result for one sample run is plotted in red in Figure $6 \mathrm{~b}$ and compared to the true spanwise position in blue. Interestingly, the $z_{s}$ trajectory corresponds very well with the $z$-trajectory especially for $x<1.5 \delta$. The small and growing deviation between the curves $1 \delta<x<2 \delta$ suggests that the particle slid in the spanwise direction while rolling over this range. Similar to this result, in most runs, the spanwise motion for sphere P3 often has a rolling component induced by a spanwise fluid torque and wall friction.

While many previous studies concluded that particles with smaller $\mathrm{St}^{+}$tend to migrate into low-speed streaks or zones, our results on larger particles show no preferential behavior of this sort. Here, a particle that lags in a surrounding slow-moving region and is approached by a trailing fast-moving region will accelerate and travel with the high momentum fluid. Similarly, a particle that lags in a surrounding fast-moving region could be overtaken by a trailing slow-moving region and then decelerate. The various runs observed do not reveal any obvious tendency of the spheres to migrate in the spanwise direction from high- to low-speed zones. We do notice examples where a particle traveling within a long, slow-moving zone as shown in Figure $3 \mathrm{~b}$, tends to meander with the coherent structure. Also, in some other runs, the particle spanwise motion is strongly affected by the spanwise fluid motion. For example, in Figure $3 \mathrm{k}$, instead of moving towards the slow-moving zone in the $+z$ direction, the sphere moves towards $-z$. By referring to the corresponding spanwise velocity contour plot shown in Figure 6r, we note that this sphere changes direction because it becomes surrounded by fluid moving in the negative spanwise direction (blue).

\section{Conclusions}

Simultaneous particle tracking and stereoscopic particle image velocimetry experiments were conducted to investigate the three-dimensional translation and rotation of spheres and their surrounding fluid motions at $R e_{\tau}=670$ and 1300. Individual spheres with diameters of 56 and 116 viscous units and specific gravities of $1.006(\mathrm{P} 1)$ and $1.152(\mathrm{P} 3)$ were released from rest and tracked over a streamwise distance up to $x \approx 5 \delta$. 
In this paper, the fluid motions surrounding the sphere within the field of view of $0.3<x / \delta<1.7$ and $-0.5<z / \delta<0.5$ at $y=0.7 d$ were discussed.

Upon release, when the mean shear lift force is larger than the net buoyancy force, the less dense sphere P1 lifts off from the wall in almost all runs. This sphere undergoes multiple lift-off events, including saltation and resuspension, with a stronger lift-off magnitude observed at higher $R e_{\tau}$. Although this sphere mostly travels above the wall, it nevertheless lags behind the mean fluid velocity even after attaining an approximate average terminal velocity. Throughout its trajectory, the lifting sphere translates with very weak or minimal rotations about any axis. By contrast, upon release, the wall-interacting sphere P3 first slides along the wall with minimal rotation. At $R e_{\tau}=670$, the initial acceleration of this sphere is significantly retarded by the opposing friction force, in contrast to the cases where the sphere accelerates steeply over a streamwise distance of $\delta$. After propagating downstream by $\approx 1.5 \delta$, this sphere accelerates again as forward rolling (with slipping) and repeated small lift-off events of magnitude less than $0.1 d$ begin to occur. Hence, wall friction is important in impeding the acceleration of the denser sphere as well as in prompting the rolling motion. A detailed discussion on sphere motions can be found in Tee et al. (2020).

By correlating the sphere and fluid motions, the current results suggested that coherent structures in the boundary layers such as the high and low momentum regions, vortex shedding, as well as spanwise fluid motions, have important effects on sphere kinematics. For both spheres, the fluctuations in the sphere streamwise velocity are strongly affected by the large-scale coherent structures that approach and move over the sphere. For sphere P1, the fluctuations in the sphere streamwise velocity are also correlated with the sphere wall-normal positions. Meanwhile, for sphere P3, the fluctuations are more correlated to the vortex shedding. In all cases, the spheres move significantly in the spanwise direction migrating up to $12 \%$ of the streamwise distance traveled. No preferential alignments with fluid structures are observed as the spheres are seen within both fast- and slow-moving zones. For the denser sphere, due to the direct wall interactions, its spanwise motion is strongly correlated with rotation about the $x$-axis which must be induced by a fluid torque. The spanwise motion of the lifting sphere, which travels mostly above the wall, correlates with spanwise motion in the fluid. A sphere that travels within a long, slow-moving zone is affected by the meandering nature of that structure. As most spheres lag the local fluid velocity, the magnitude of the relative velocity plays an important role in determining whether the sphere travels a significant distance within one zone or whether it is overcome relatively rapidly by succeeding fast- and slow-moving zones.

\section{Acknowledgements}

The authors thank Diogo C. Barros, Nicholas Morse, Ben Hiltbrand and Alessio Gardi for their help with this project. This work was funded by the U.S. National Science Foundation (CBET-1510154). The first author is supported by the University of Minnesota Graduate School under the Doctoral Dissertation Fellowship.

\section{References}

Baker LJ and Coletti F (2021) Particle-fluid-wall interaction of inertial spherical particles in a turbulent boundary layer. J Fluid Mech 908

Barros D, Hiltbrand B, and Longmire EK (2018) Measurement of the translation and rotation of a sphere in fluid flow. Exp Fluids 59:104

Bellani G, Byron ML, Collignon AG, Meyer CR, and Variano EA (2012) Shape effects on turbulent modulation by large nearly neutrally buoyant particles. J Fluid Mech 712:41-60

Costa P, Brandt L, and Picano F (2020) Interface-resolved simulations of small inertial particles in turbulent channel flow. J Fluid Mech 883

Crowe CT (2005) Multiphase flow handbook. CRC press

Dennis DJ and Nickels TB (2011) Experimental measurement of large-scale three-dimensional structures in a turbulent boundary layer. Part 2. Long structures. J Fluid Mech 673:218

Drake TG, Shreve RL, Dietrich WE, Whiting PJ, and Leopold LB (1988) Bedload transport of fine gravel observed by motion-picture photography. J Fluid Mech 192:193-217 
Ebrahimian M, Sanders RS, and Ghaemi S (2019) Dynamics and wall collision of inertial particles in a solid-liquid turbulent channel flow. J Fluid Mech 881:872-905

Francis J (1973) Experiments on the motion of solitary grains along the bed of a water-stream. Proceedings of the Royal Society of London A Mathematical and Physical Sciences 332:443-471

Hall D (1988) Measurements of the mean force on a particle near a boundary in turbulent flow. J Fluid Mech 187:451-466

Kabsch W (1976) A solution for the best rotation to relate two sets of vectors. Acta Crystallographica Section A: Crystal Physics, Diffraction, Theoretical and General Crystallography 32:922-923

Kaftori D, Hetsroni G, and Banerjee S (1995) Particle behavior in the turbulent boundary layer. I. Motion, deposition, and entrainment. Phys Fluids 7:1095-1106

Klein S, Gibert M, Bérut A, and Bodenschatz E (2013) Simultaneous 3D measurement of the translation and rotation of finite-size particles and the flow field in a fully developed turbulent water flow. Measurement Science and Technology 24:024006

Mathai V, Neut MW, van der Poel EP, and Sun C (2016) Translational and rotational dynamics of a large buoyant sphere in turbulence. Exp Fluids 57:51

Niño Y and Garcia M (1996) Experiments on particle-turbulence interactions in the near-wall region of an open channel flow: implications for sediment transport. J Fluid Mech 326:285-319

Pedinotti S, Mariotti G, and Banerjee S (1992) Direct numerical simulation of particle behaviour in the wall region of turbulent flows in horizontal channels. Int J Multiph Flow 18:927-941

Rashidi M, Hetsroni G, and Banerjee S (1990) Particle-turbulence interaction in a boundary layer. Int J Multiph Flow 16:935-949

Sciacchitano A, Scarano F, and Wieneke B (2012) Multi-frame pyramid correlation for time-resolved PIV. Exp Fluids 53:1087-1105

Sumer BM and Oguz B (1978) Particle motions near the bottom in turbulent flow in an open channel. $J$ Fluid Mech 86:109-127

Sutherland AJ (1967) Proposed mechanism for sediment entrainment by turbulent flows. J Geophys Res 72:6183-6194

Tan YM and Longmire EK (2017) Recovery of vortex packet organization in perturbed turbulent boundary layers. Phys Rev Fluids 2:104602

Tee YH, Barros DC, and Longmire EK (2020) Motion of finite-size spheres released in a turbulent boundary layer. Int J Multiph Flow 133:103462

van Hout R (2013) Spatially and temporally resolved measurements of bead resuspension and saltation in a turbulent water channel flow. J Fluid Mech 715:389-423

van Hout R, Eisma J, Elsinga GE, and Westerweel J (2018) Experimental study of the flow in the wake of a stationary sphere immersed in a turbulent boundary layer. Phys Rev Fluids 3:024601

Westerweel J and Scarano F (2005) Universal outlier detection for PIV data. Exp Fluids 39:1096-1100

Wu Y and Christensen K (2006) Population trends of spanwise vortices in wall turbulence. J Fluid Mech 568:55-76

Yousefi A, Costa P, and Brandt L (2020) Single sediment dynamics in turbulent flow over a porous bedinsights from interface-resolved simulations. J Fluid Mech 893:A24, 1-28

Zeng L, Balachandar S, Fischer P, and Najjar F (2008) Interactions of a stationary finite-sized particle with wall turbulence. J Fluid Mech 594:271-305

Zimmermann R, Gasteuil Y, Bourgoin M, Volk R, Pumir A, and Pinton JF (2011) Tracking the dynamics of translation and absolute orientation of a sphere in a turbulent flow. Rev. Sci. Inst. 82:033906 